\title{
Atividade eletromiográfica dos músculos estabilizadores da escápula durante variações do exercício push up em indivíduos com e sem síndrome do impacto do ombro
}

\author{
Electromyographic activity of scapular stabilizers muscles during push up \\ exercise variations in subjects with and without shoulder impingement \\ syndrome
}

\author{
L.P. Batista, V.A. Oliveira, A.T. Pirauá, A.R. Pitangui, R.C. Araújo
}

ARTIGO ORIGINAL | ORIGINAL ARTICLE

\begin{abstract}
Exercícios em cadeia cinética fechada (CCF) são comumente empregados na reabilitação da Síndrome do Impacto do Ombro (SIO), condição dolorosa comum em atletas, relacionada ao desequilíbrio muscular entre os estabilizadores da escápula. O objetivo do estudo foi comparar a atividade eletromiográfica (EMG) do serrátil anterior (SA), trapézio superior (TS), trapézio médio (TM) e trapézio inferior (TI) e a razão EMG destes músculos durante variações do exercício push up em indivíduos assintomáticos e com SIO. Foram avaliados 30 voluntários divididos em dois grupos: controle e SIO. A atividade EMG foi registrada durante a realização do knee push up (KPU) e one-arm knee push up (OKP), em base instável e estável de apoio. Para inferência estatística foi utilizada a análise de variância (ANOVA), pós teste Tukey, com nível de significância de 5\%. A superfície instável acarretou diminuição na ativação do SA nos dois grupos e aumento da razão TS/SA $(p<.03)$ no grupo SIO. Os resultados do estudo evidenciaram diferentes respostas de ativação muscular nos indivíduos assintomáticos e com SIO frente a condições que alteram a estabilidade da base de apoio.

Palavras-chave: dor de ombro, eletromiografia, terapia por exercício
\end{abstract}

ABSTRACT

Closed Kinetic Chain exercises (CKC) are commonly employed in the rehabilitation of Shoulder Impingement Syndrom (SIS), painful condition common in athletes, related to muscle imbalance between the scapular stabilizers. The objectives of this study was to compare the electromyographic activity (EMG) of the serratus anterior (SA), upper trapezius (UT), middle trapezius (MT) and lower trapezius (LT) and the electromyographic ratio during exercises push up in asyntomatic individuals and SIS. Thirty volunteers divided into two groups: control and SIS. The EMG activity was recorded during the knee push up (KPU) and one-arm knee push up (OKP), on stable and unstable bases of support. For statistical inference was used an analysis of variance (ANOVA) and Tukey post-test, with significance level of 5\%. The unstable surface provided in decreased activation of SA in both groups and increased ratios of UT / SA $(p<.03)$ in the SIS. The results of the study showed different responses of muscle activation, in individuals with asymptomatic and SIS, face to conditions that alter the stability of the base of support.

Keywords: shoulder pain, electromyography, exercise therapy

Submetido: 29.06.2012 | Aceite: 15.05.2013

Laísla da Silva Paixão Batista, Valéria Mayaly Alves de Oliveira, Ana Carolina Rodarti Pitangui, Rodrigo Cappato de Araújo. Universidade de Pernambuco, Petrolina, PE, Brasil.

André Luiz Torres Pirauá. Universidade de Pernambuco, Recife, Brasil.

Endereço para correspondência: Rodrigo Cappato de Araújo, Universidade de Pernambuco, Campus Petrolina, Departamento de Fisioterapia, BR 203 Km 2 S/N, Vila Eduardo, CEP 56300-000, Petrolina, PE, Brasil.

E-mail: rodrigocappato@yahoo.com.br 
A adequada ativação dos músculos estabilizadores escapulares, principalmente o serrátil anterior e o trapézio, é fundamental ao movimento coordenado das articulações escapulotorácica e glenoumeral, e contribui para efetiva função do ombro (Lukasiewicz, McClure, Michener, Pratt, \& Sennet, 1999; McClure, Michener, Sennett, \& Karduna, 2001). Assim o desequilíbrio muscular e as alterações no sincronismo destas articulações podem determinar condições dolorosas, como a Síndrome do Impacto do Ombro (SIO), uma das principais afecções musculoesqueléticas em praticantes de modalidades esportivas que exigem o movimento do braço acima da cabeça(Ludewig \& Braman, 2011; Phadke, Camargo, \& Ludewig, 2009).

A SIO é definida como a condição dolorosa decorrente do impacto mecânico e consequente compressão das estruturas contidas no espaço subacromial, sobretudo durante a elevação do braço, geralmente relacionada ao uso excessivo da articulação glenoumeral (Neer, 1983). Entre os diferentes fatores relacionados à etiologia desta síndrome estão as alterações na ativação dos músculos estabilizadores escapulares, caracterizadas pelo aumento da ativação do músculo trapézio superior em relação ao serrátil anterior, que ocasiona um aumento da translação superior da escápula e diminuição da rotação superior e tipping escapular, resultando na redução do espaço subacromial e predispondo o impacto (Kibler, 1998; Kibler et al., 2002; Ludewig \& Cook, 2000).

Dessa forma, os exercícios em cadeia cinética fechada (CCF) têm sido empregados nos programas de reabilitação, visando à ativação do músculo serrátil anterior e das diferentes porções do músculo trapézio na tentativa de alcançar o equilíbrio destas forças musculares e com isso adequação da cinemática escapular (Kibler \& Livingston, 2011; Tucker, Armstrong, Gribble, Timmons, \& Yeasting, 2010). Estes exercícios auxiliam na recuperação da estabilidade dinâmica da articulação glenoumeral, uma vez que envolvem mecanismos neuromusculares e proprioceptivos, que possibilitam a coativação da musculatura estabilizadora desta articulação (Dillman, Murray, \& Hintermeister, 1994; Lephart \& Henry, 1996), sendo eficazes para aquisição e manutenção de uma base estável para os diversos movimentos do ombro.

Assim, a atividade eletromiográfica (EMG) dos músculos serrátil anterior e trapézio têm sido avaliada, por meio da eletromiografia de superfície, durante a realização de exercícios de push up e suas variações, na tentativa de identificar o padrão de ativação muscular durante estes exercícios, principalmente quando incluídas mudanças na instabilidade da superfície de apoio (Ludewig, Hoff, Osowski, Meschke, \& Rundquist, 2004; Martins et al., 2008; Oliveira, De Morais, \& De Brum, 2008; Park \& Yoo, 2011). Estes estudos têm demonstrado que os exercícios em CCF proporcionam níveis significativos de ativação muscular dos estabilizadores escapulares, possibilitando inclusive maior ativação do serrátil anterior em relação ao músculo trapézio fibras superiores, fato que seria fundamental na reabilitação dos indivíduos com a SIO (Park \& Yoo, 2011; Tucker, Campbell, Swartz, \& Armstrong, 2008).

Considerando a existência de desequilíbrio muscular entre os estabilizadores escapulares nos indivíduos com a SIO, e os efeitos dos exercícios em CCF relatados anteriormente, é importante avaliar a necessidade da inserção destes exercícios associados à base instável de apoio na reabilitação, uma vez que nessa condição seria requisitada uma melhor estratégia neuromotora para garantir a estabilização do membro superior e tronco (Ludewig et al., 2004).

Por outro lado, durante o processo de reabilitação é necessário implementar um programa de exercícios específicos para cada etapa de tratamento, respeitando principalmente a evolução clínica de cada paciente. Diante disso, observa-se a busca pela prescrição de tarefas isométricas, consideradas mais simples e mais seguras do ponto de vista da execução, e posteriormente a evolução para a realização das taferas de forma 
dinâmica. Assim o objetivo deste estudo foi comparar a atividade eletromiográfica do serrátil anterior e do trapézio superior, médio e inferior, assim como a razão entre atividade EMG destes músculos durante a realização isométrica de variações do push up em indivíduos assintomáticos e com síndrome do impacto do ombro.

O presente estudo tem como hipótese que a inserção da superfície instável durante as tarefas isométricas, consideradas mais simples, é capaz de proporcionar alterações na atividade EMG sem que ocorra alteração na proporção de ativação dos músculos trapézio e serrátil anterior, nos indivíduos assintomáticos. Por outro lado, se espera que os indivíduos com SIO, apesar de fisicamente ativos, apresentem alterações na proporção de ativação desses músculos como estratégia adaptativa frente a instabilidade criada.

\section{MÉTODO}

\section{Amostra}

Foram avaliados 30 voluntários, do sexo masculino, praticantes de atividades físicas que envolvessem movimentos repetidos do membro superior acima da cabeça: voleibol (dois), natação (dois), lutas (dez) ou musculação (dezesseis), há pelo menos seis meses, com frequência mínima de quatro vezes por semana e duração semanal superior à seis horas de treino. Os voluntários foram divididos em dois grupos com igual número de participantes: o primeiro foi composto por indivíduos que apresentassem sinais e sintomas e/ou diagnóstico confirmado da SIO (grupo SIO) e o outro compreendia indivíduos assintomáticos (grupo controle). Os dados antropométricos e domi- nância funcional dos dois grupos estão apresentados na tabela 1.

Os critérios de inclusão do estudo foram: sujeitos do sexo masculino, fisicamente ativos, com idade entre 18 e 35 anos. Além disso, para distribuição entre os grupos, foram estabelecidos alguns critérios. No grupo SIO, foram incluídos sujeitos que apresentaram dor na articulação do ombro (membro dominante) por pelo menos seis semanas anteriores à avaliação, que referiram dor à palpação e apresentaram positividade em pelo menos dois dos três testes clínicos específicos avaliados (Neer, Jobe e Hawkins) (Hawkins \& Kennedy, 1980; Jobe \& Moynes, 1982; Neer, 1983) ou aqueles que tivessem confirmado o diagnóstico clínico de SIO. Os demais foram incluídos no grupo controle.

Foram excluídos do estudo, os voluntários que apresentaram histórico de cirurgias e fraturas na cintura escapular, ombro e cervical, lesão nervosa periférica, doenças articulares degenerativas. Todos os voluntários assinaram o termo de Consentimento Livre e Esclarecido, segundo a resolução n 196/96 do Conselho Nacional da Saúde e o estudo foi aprovado pelo Comitê de Ética em Pesquisa da Universidade de Pernambuco sob o protocolo 274/10.

\section{Instrumentos}

Para aquisição dos registros eletromiográficos dos músculos serrátil anterior (SA), trapézio fibras superiores (TS), inferiores (TI) e médias (TM), foram utilizados quatro canais do sistema Myosystem Br-1 da Datahominis Tecnologia Ltda ${ }^{\circledR}$ (Uberlândia - Brasil). Os sinais mioelétricos foram adquiridos por meio de eletrodos de superfícies ativos, diferenciais de ganho de 20

Tabela 1

Dados Antropométricos e dominância funcional dos indivíduos do grupo Controle e Síndrome do Impacto do Ombro (SIO)

\begin{tabular}{ccc}
\hline Característica & SIO $(n=15)$ & Controle $(n=15)$ \\
\hline Idade (anos) & $22.00 \pm 3.87$ & $20.73 \pm 2.87$ \\
Massa Corporal (quilogramas) & $73.3 \pm 8.57$ & $76.90 \pm 12.86$ \\
Estatura (metros) & $1.76 \pm 0.08$ & $1.76 \pm 0.07$ \\
Dominância Direita & $14(93.3 \%)$ & $14(93.3 \%)$ \\
Dominância Esquerda & $1(6.7 \%)$ & $1(6.7 \%)$ \\
\hline
\end{tabular}


vezes, compostos por duas barras retangulares paralelas de prata pura $(10 \times 2 \times 1 \mathrm{~mm}$, com distância de $10 \mathrm{~mm}$ entre barras), da Datahominis Tecnologia Ltda ${ }^{\circledR}$ (Uberlândia - Brasil). $\mathrm{O}$ equipamento possuía aterramento e aquisição simultânea comum para os canais, filtro de banda $10 \mathrm{~Hz}$ a $5 \mathrm{KHz}$; três estágios de amplificação, impedância dos canais de $10 \mathrm{G} \Omega$ em modo diferencial, Razão de Rejeição em Modo Comum de 92 dB, 16 bits e faixa resolução dinâmica, faixa de amplitude de $-10 \mathrm{~V}$ a $+10 \mathrm{~V}$ e placa conversora analógica-digital.

Antes da colocação dos eletrodos foram realizadas a tricotomia, leve abrasão e assepsia da pele com álcool, para diminuir a impedância da pele e eliminar possíveis interferências. O posicionamento dos eletrodos nos músculos Trapézio Superior (TS), Trapézio Médio (TM) e Trapézio Inferior (TI) foi feito de acordo com as recomendações do projeto SENIAM (Hermens, Freiks, \& Merletti, 1999). Para o músculo serrátil anterior o eletrodo foi posicionado sobre a quinta costela, segundo descrição de Hintermeister, Lange, Schultheis, Bey, \& Hawkins (1998). Todos os eletrodos foram fixados com esparadrapo. O eletrodo de referência foi posicionado no punho, sobre o manúbrio esternal e interposto com gel eletrocondutor. Para os voluntários do grupo controle, os eletrodos foram posicionados no membro dominante.

Para o processamento dos dados eletromiográficos, foi utilizado o programa Myosystem Br-1 versão 3.5.4. O sinal eletromiográfico bruto foi retificado, feito o alisamento com filtro butterworth de $4^{\mathrm{a}}$ e $10 \mathrm{~Hz}$ ordem, e utilizado para derivar os valores da amplitude eletromiográfica obtidos por meio do cálculo do root mean square (RMS). Os dados foram coletados a $4000 \mathrm{~Hz}$ e aplicados filtros digitais de passa baixa de $500 \mathrm{~Hz}$ e passa alta de $15 \mathrm{~Hz}$. Os valores RMS EMG foram normalizados pelo valor máximo da amplitude eletromiográfica obtida nas três repetições da contração voluntária máxima (CVM).

A proporção de ativação muscular entre os músculos trapézio superior e o inferior (TS/TI) e o trapézio superior e serrátil anterior (TS/SA) em cada tarefa, pôde ser avaliada por meio do cálculo da razão eletromiográfica. Neste procedimento também se realizou a normalização dos dados brutos, sendo calculada por meio da razão entre a média RMS obtida em cada exercício e o valor de RMS máximo encontrado na CVM de cada músculo. Assim, as razões EMG foram mensuradas pela razão do valor normalizado do TS dividido pelo valor normalizado da razão SA e TI. Identificando assim, qual músculo sobressaia-se mais em cada tarefa. O valor de razão foi considerada baixo se fosse menor que 0.3 , que indicava uma ativação do SA ou TI três vezes maior em relação ao TI ou TS. Os valores da razão próximo ou maior que 1.0 foram considerados como ativação muscular similar entre os dois músculos ou maior predominância do TS sobre o SA e TI.

\section{Procedimentos}

Inicialmente foram realizados a anamnese para coleta de dados pessoais e antropométricos e o exame físico, no qual foram realizados os testes clínicos de Neer, Jobe e Hawkins para confirmação da SIO e dessa maneira realizar a distribuição dos voluntários nos dois grupos. Concluída a avaliação, foi realizado um sorteio que determinou a ordem de execução das tarefas e posteriormente foi feita a limpeza da pele com álcool e a colocação dos eletrodos.

Em seguida, foram realizadas três repetições de uma contração voluntária máxima (CVM) para cada um dos músculos avaliados, de acordo com o posicionamento do teste muscular manual (Kendall, McCreary, \& Provence, 1995). Estas deveriam ser mantidas por 6 segundos, com intervalos de 2 minutos entre cada repetição. $O$ valor máximo da amplitude registrado nas CVMs foi utilizado para a normalização do sinal EMG.

Finalizada a última CVM, foram dadas orientações para realização das tarefas: knee push up (KPU) e one - arm knee push up (OKP). 
Para a realização do knee push up, os voluntários deveriam ficar em posição de quatro apoios mantendo $90^{\circ}$ de flexão de quadril e joelho, tronco alinhado e cabeça em posição neutra. Os membros superiores deveriam estar a $90^{\circ}$ de flexão do ombro, cotovelos em completa extensão e punhos a $90^{\circ}$ de extensão. As mãos deveriam estar posicionadas na base de suporte a uma distancia equivalente a largura do ombro (Figura 1). Para o one-arm push up o posicionamento foi semelhante, apenas foi solicitado aos voluntários que colocassem o membro superior contralateral na região dorsal (Figura 2). Os voluntários foram orientados a manter essa posição, segundo o comando verbal do pesquisador. Um disco inflável proprioceptivo modelo DiscoFlex - Mercur S.A (Santa Cruz do Sul, Brasil) foi utilizado para conferir a instabilidade à base de suporte. Em todas as situações foi realizada a medição e marcação dos pontos para o posicionamento das mãos.

Foram realizadas três repetições de cada exercício, em base estável e instável. As tarefas foram mantidas por 8 segundos, com intervalos de 1,5 minutos entre as repetições e os exercícios, para minimizar possíveis efeitos de fadiga local. A ordem da realização dos exercícios foi determinada por sorteio.

\section{Análise Estatística}

Para processamento e análise dos dados foi utilizado o programa SPSS versão 16.0. Após consolidação e validação dos dados inseridos, foi utilizado teste de Shapiro-Wilk para a avaliação da normalidade dos dados. Inicialmente, foi aplicado o teste $t$ de student para comparação intergrupos, das variáveis idade, estatura e massa corporal. O efeito dos grupos, exercícios e a interação dessas variáveis sobre a atividade EMG músculos foi avaliado pela

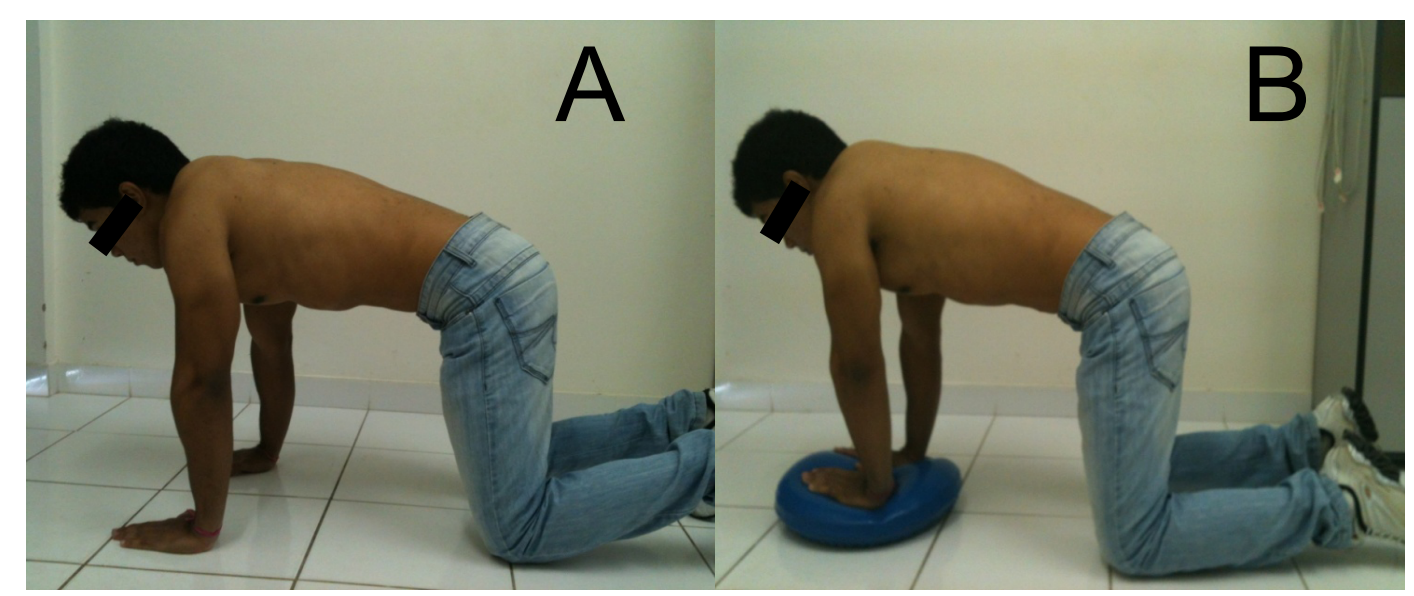

Figura 1. Posicionamento para a realização do Knee push up em base estável (A) e instável (B)

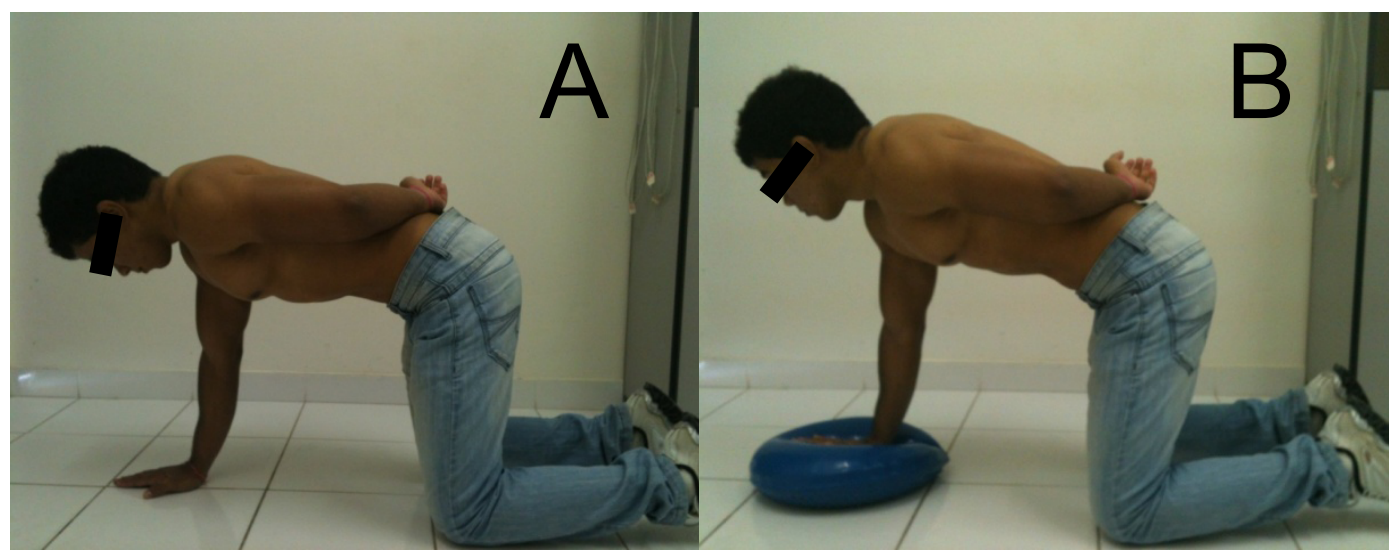

Figura 2. Posicionamento para a realização do One arm Knee push up em base estável (A) e instável (B) 
análise de variância ANOVA ( 2 grupos x 4 exercícios x 4 músculos) com pós teste de Tukey. Em todas as situações foi utilizado nível de significância de $5 \%$. Além disso, nas variáveis em que foram observados valores de $p<$ .05 , foi calculado eta quadrado $\left(\eta^{2}=\right.$ efeito da variância/ variância total) para avaliação da magnitude do efeito.

\section{RESULTADOS}

\section{Características da amostra}

A análise intergrupos não revelou diferenças estatísticas para as variáveis idade $(p=.873)$, massa corporal $(p=.902)$ e estatura $(p=.981)$ (Tabela 1).

\section{Atividade EMG}

$\mathrm{Na}$ tabela 2 estão descritos os valores da atividade EMG dos músculos avaliados, nos diferentes grupos e exercícios. A análise de variância evidenciou nenhum efeito dos grupos na atividade EMG dos músculos TS $\left[\mathrm{F}_{(4.195)}=\right.$ $0.515 ; p=.479]$, TM $\left[\mathrm{F}_{(4.195)}=0.016 ; p=.90\right]$, TI $\left[\mathrm{F}_{(4.195)}=0.175 ; p=.679\right]$ e SA $\left[\mathrm{F}_{(4.195)}=\right.$ 0.271; $p=.607]$. Também não foi observada interação entre grupos e diferentes exercícios para os valores EMG dos músculos TS $\left[\mathrm{F}_{(4.195)}\right.$

$$
\begin{aligned}
& =2.248 ; p=.097], \mathrm{TM}\left[\mathrm{F}_{(4.195)}=1.164 ; p\right. \\
& =.328], \mathrm{TI}\left[\mathrm{F}_{(4.195)}=0.937 ; p=.427\right] \text { e SA } \\
& {\left[\mathrm{F}_{(4.195)}=0.395 ; p=.757\right] .}
\end{aligned}
$$

No entanto, para o grupo controle foi verificado que o tipo de exercício apresentou efeito sobre a atividade do músculo SA $\left[\mathrm{F}_{(2.713)}=10.515 ; p=.003 ; \eta^{2}=.38\right]$. Nesse caso foi possível observar que a superfície instável foi responsável pela diminuição da atividade do SA, no grupo controle, em relação à superfície estável, nos exercícios KPU ( $p=$ .02) e OKP $(p=.03)$. Para o grupo SIO, foi observado efeito do tipo de exercício sobre a atividade dos músculos TI $\left[\mathrm{F}_{(2.713)}=6.766 ; p=\right.$ $\left..001 ; \eta^{2}=.25\right]$ e $S A\left[\mathrm{~F}_{(2.713)}=10.515 ; p=.003\right.$; $\left.\eta^{2}=.38\right]$. Foram observados menores valores EMG no exercício OKP instável em relação ao KPU estável, tanto para o TI $(p=.01)$, quanto para o SA $(p=.001)$. Nos demais músculos não se evidenciou efeito dos diferentes tipos de exercício sobre a atividade EMG.

Referente à comparação dos níveis de atividade EMG dos quatro músculos em cada exercício, foi possível observar, no grupo SIO, nenhuma diferença estatística nos exercícios KPU estável $\left[\mathrm{F}_{(2.760)}=2.150 ; p=.104\right]$, KPU instável $\left[\mathrm{F}_{(2.760)}=1.053 ; p=.376\right]$, OKP

Tabela 2

Registros eletromiográficos normalizados (\% CVM) dos músculos Trapézio Superior (TS), Trapézio Médio (TM), Trapézio

\begin{tabular}{|c|c|c|c|c|}
\hline \multicolumn{5}{|c|}{ KPU } \\
\hline & \multicolumn{2}{|c|}{ SIO } & \multicolumn{2}{|c|}{ Controle } \\
\hline & Estável & Instável & Estável & Instável \\
\hline TS & $17.1 \pm 21.2$ & $19.2 \pm 19.1$ & $10.3 \pm 6.9$ & $13.5 \pm 11.1$ \\
\hline $\mathrm{TM}$ & $23.6 \pm 26.7$ & $15.3 \pm 22.7$ & $28.3 \pm 36.6$ & $23.8 \pm 24.2$ \\
\hline $\mathrm{TI}$ & $32.9 \pm 32.1^{\mathrm{d}}$ & $19.8 \pm 39.5$ & $22.7 \pm 18.0$ & $18.3 \pm 17.4$ \\
\hline SA & $38.3 \pm 18.4$ & $30.7 \pm 14.2$ & $44.5 \pm 22.1^{\mathrm{a}, \mathrm{f}}$ & $30.3 \pm 21.7^{f}$ \\
\hline \multicolumn{5}{|c|}{ OKP } \\
\hline & \multicolumn{2}{|c|}{ SIO } & \multicolumn{2}{|c|}{ Controle } \\
\hline & Estável & Instável & Estável & Instável \\
\hline TS & $15.9 \pm 16.3$ & $13.6 \pm 10.3$ & $15.8 \pm 13.4$ & $13.9 \pm 8.0$ \\
\hline $\mathrm{TM}$ & $26.8 \pm 28.5$ & $20.5 \pm 26.1$ & $22.7 \pm 18.6$ & $19.7 \pm 13.8$ \\
\hline $\mathrm{TI}$ & $16.9 \pm 16.6$ & $10.0 \pm 12.4^{\mathrm{d}}$ & $14.6 \pm 11.6$ & $12.7 \pm 12.1$ \\
\hline SA & $40.0 \pm 11.2^{\mathrm{e}}$ & $21.4 \pm 10.4^{\mathrm{e}}$ & $41.9 \pm 23.9^{\mathrm{a}, \mathrm{b}, \mathrm{c}, \mathrm{e}}$ & $26.1 \pm 16.4^{\mathrm{c}, \mathrm{e}}$ \\
\hline
\end{tabular}
Inferior (TI) e Serrátil Anterior (SA), durante a tarefa Knee push up (KPU) e o One-arm push-up (OKP) nos grupos Síndrome do Impacto (SIO) e Controle.

$p<.05$; (a)indica diferença estatística entre TS e SA, (b) entre SA e TM e (c) entre SA e TI.

(d) Indica diferença do TI entre KPU estável e OKP instável; (e) Indica diferença do SA entre OKP estável e instável; (f) Indica diferença do SA entre KPU estável e Instável. 
estável $\left[\mathrm{F}_{(2.760)}=2.590 ; p=.061\right]$ e OKP instável $\left[\mathrm{F}_{(2.760)}=1.101 ; p=.356\right]$. No grupo controle, a análise de variância identificou diferenças na atividade EMG entre os músculos no exercício KPU estável $\left[\mathrm{F}_{(2.760)}=4.398 ; p\right.$ $\left.=.001 ; \eta^{2}=.20\right]$, com maior atividade do SA em relação ao TS $(p=.001)$. Por outro lado, no exercício KPU instável não foram observadas diferenças significativas $\left[\mathrm{F}_{(2.760)}=2.212 ; p=\right.$ .096]. No exercício OKP instável foi evidenciada diferença significativa na atividade EMG entre os músculos $\left[\mathrm{F}_{(2.760)}=3.505 ; p=.021 ; \eta^{2}\right.$ $=.16]$, com maior atividade do SA comparado ao músculo TI $(p=.04)$. Por fim, no exercício OKP estável se observou um maior número de diferenças estatísticas $\left[\mathrm{F}_{(2.760)}=8.199 ; p=\right.$ $.001 ; \eta^{2}=.32$ ]. Nesse casso, a atividade EMG do músculo SA foi superior em relação ao TS ( $p$ $=.001)$, TM $(p=.04)$ e ao TI $(p=.001)$.

\section{Razão TS/SA e TS/TI}

$\mathrm{Na}$ tabela 3 estão descritos os valores das razões TS/SA e TS/TI, nos diferentes grupos e exercícios. A análise de variância evidenciou nenhum efeito dos grupos nas razões TS/SA $\left[\mathrm{F}_{(4.195)}=0.035 ; p=.853\right]$ e TS/TI $\left[\mathrm{F}_{(4.195)}=\right.$ $1.171 ; p=.288]$. Também não foi observada interação entre grupos e diferentes exercícios para os valores das razões TS/SA $\left[\mathrm{F}_{(4.195)}=\right.$ $0.223 ; p=.640]$ e TS/TI $\left[\mathrm{F}_{(4.195)}=0.262 ; p=\right.$ $.613]$.
No entanto, a razão TS/SA foi influenciada pelo tipo de exercício $\left[\mathrm{F}_{(4.195)}=4.497 ; p=.04\right.$; $\left.\eta^{2}=.10\right]$. Nesse caso, a superfície instável foi responsável pelo aumento significativo dessa variável durante a execução dos exercícios KPU $(p=.03)$ e OKP $(p=.03)$, no grupo SIO. Entretanto, para os valores da razão TS/TI não foi verificado nenhum efeito do tipo de exercício $\left[\mathrm{F}_{(4.195)}=1.105 ; p=.302\right]$.

No grupo SIO, os resultados mostraram que a razão TS/TI apresentou valores superiores à razão TS/SA durante a realização dos exercícios OKP estável $(p=.038)$, OKP instável ( $p$ $=.013)$ e KPU instável $(p=.048)$. No entanto, nenhuma diferença foi observada entre as razões no exercício KPU estável $(p=.295)$. No grupo controle não foram observadas diferenças entre as razões no exercício KPU realizado em ambas as superfícies de apoio (estável $-p=.120$; instável $-p=.164$ ). No entanto, no exercício OKP, em ambas as superfícies, foram observados maiores valores da razão TS/TI em comparação à razão TS/SA (estável $-p=.012$; instável $-p=.004)$. (Tabela 3).

\section{DISCUSSÃO}

O objetivo do presente estudo foi avaliar e comparar a atividade muscular dos estabilizadores da escápula entre praticantes de atividade física com e sem histórico de síndrome do impacto durante quatro exercícios de cadeia

Tabela 3

Razões eletromiográficas normalizadas (\% CVM) durante a tarefa Knee push up (KPU) e o One-arm knee push-up (OKP) nos grupos Síndrome do Impacto (SIO) e Controle

\begin{tabular}{|c|c|c|c|c|}
\hline \multicolumn{5}{|c|}{ KPU } \\
\hline & \multicolumn{2}{|c|}{ SIO } & \multicolumn{2}{|c|}{ Controle } \\
\hline & Estável & Instável & Estável & Instável \\
\hline $\mathrm{TS} / \mathrm{SA}$ & $0.52 \pm 0.68^{b}$ & $0.78 \pm 0.90^{\mathrm{a}}$ & $0.40 \pm 0.37$ & $0.69 \pm 0.80$ \\
\hline $\mathrm{TS} / \mathrm{TI}$ & $1.54 \pm 3.63^{\mathrm{b}}$ & $3.46 \pm 2.95$ & $0.90 \pm 1.16$ & $1.09 \pm 0.75$ \\
\hline \multicolumn{5}{|c|}{ OKP } \\
\hline & \multicolumn{2}{|c|}{$\mathrm{SIO}$} & \multicolumn{2}{|c|}{ Controle } \\
\hline & Estável & Instável & Estável & Instável \\
\hline $\mathrm{TS} / \mathrm{SA}$ & $0.36 \pm 0.29^{\mathrm{a}, \mathrm{b}}$ & $0.77 \pm 0.59^{a}$ & $0.51 \pm 0.54^{\mathrm{a}}$ & $0.68 \pm 0.53^{\mathrm{a}}$ \\
\hline TS/TI & $2.25 \pm 3.36$ & $2.27 \pm 2.14$ & $1.61 \pm 1.19$ & $1.70 \pm 1.15$ \\
\hline
\end{tabular}

$p<.05$; (a)indica diferença estatística entre TS/SA e TS/TI, (b) Indica diferença entre as superfícies estável e instável. 
cinética fechada. Vários autores (Andrade, Araújo, Tucci, Martins, \& Oliveira, 2011; Araújo, Andrade, Tucci, Martins, \& Oliveira, 2011; Ludewig et al., 2004; Martins et al., 2008; Oliveira et al., 2008; Tucker et al., 2010) têm avaliado e sugerido a inserção de exercícios de cadeia cinética fechada, tais como push up, em programas de reabilitação com o objetivo de fortalecimento dos músculos escapulares, em especial o serrátil anterior.

A busca pelo fortalecimento e reequilíbrio de forças entre os músculos serrátil anterior e trapézio se justifica, pois indivíduos com SIO geralmente apresentam aumento da atividade do trapézio fibras superiores e diminuição da atividade do serrátil anterior e alteração da cinemática escapular (Ludewig \& Cook, 2000; Ludewig et al., 2004). No presente estudo observou-se que a atividade desses músculos variou conforme o tipo de exercício e a base de apoio.

Os resultados evidenciaram, no grupo controle, maior atividade do músculo SA em relação ao TS nas tarefas realizadas sobre base estável. No entanto, o mesmo comportamento não foi observado no grupo SIO, não existindo diferença estatística na atividade do SA em relação aos demais músculos.

Em ambos os grupos foi possível observar que a realização do exercício com apoio unilateral sobre a superfície instável proporcionou a diminuição significativa da atividade do $\mathrm{SA}$, dados esses, que corroboram os estudos de Oliveira, De Morais, \& De Brum (2008) e Tucker, Armstrong, Gribble, Timmons, \& Yeasting (2010). Entretanto, Araújo, Andrade, Tucci, Martins, \& Oliveira (2011) ao avaliarem o efeito da superfície instável na atividade EMG de músculos do membro superior durante realização do exercício push up, não observaram diferenças na ativação do músculo SA em resposta a instabilidade.

Essas divergências podem estar relacionadas ao fato do estudo de Araújo et al (2011) ter controlado a carga exercida durante os exercícios, diferentemente do presente estudo e das pesquisas anteriores (Oliveira et al., 2008; Tucker et al., 2010). O controle da carga por meio do feedback sonoroso utilizado no estudo de Araújo et al (2011) parece ter contribuído para que os voluntários mantivessem níveis similares de ativação do SA. Por outro lado, os voluntários do presente estudo podem ter apenas se concentrado na manutenção do equilíbrio postural e inconscientemente podem ter descuidado em relação ao nível de esforço exercido, independente do comando verbal aplicado.

A instabilidade gerada na superfície de apoio durante os exercícios acarreta mudanças na ativação dos músculos escapulares para garantir estabilidade ao ombro (Andrade et al., 2011; Lehman, Gillas, \& Patel, 2008; Tucci et al., 2011). Conforme observado no presente estudo, nos indivíduos assintomáticos, a ativação do SA, considerado o principal estabilizador da escápula, foi superior a dos demais músculos durante OKP estável, assim como, superior à ativação do TS no KPU estável e instável e superior ao TI no OKP instável. Este pode ser considerado um mecanismo normal e necessário à sustentação e execução das tarefas solicitadas, bem como à manutenção da estabilidade do complexo articular do ombro. $\mathrm{O}$ fato de que esse comportamento não tenha sido observado nos indivíduos com a SIO pode sugerir a presença de um desequilíbrio de forças entre os músculos escapulares, em especial o SA, e sugere que as estratégias motoras adotadas nesse grupo não sejam suficientes para prover adequada estabilidade à articulação (Cools et al., 2007; De Morais Faria, Teixeira-Salmela, De Paula Goulart, \& De Souza Moraes, 2008; Hardwick, Beebe, McDonnell, \& Lang, 2006).

Em relação às razões EMG, foi observado maior valor da razão TS/TI em relação à TS/ SA no grupo controle durante o OKP estável e instável. Ressaltando que não foram observadas diferenças na ativação do TS ou TI nestes exercícios, não sendo possível determinar, se a razão elevada deve-se a maior ativação do TS ou diminuição do TI. Entretanto, foi observada 
maior ativação do SA em relação aos demais músculos neste exercício, fato que pode justificar os menores valores para razão EMG entre este músculo e o TS. Ainda no grupo controle foi possível observar que a superfície instável não alterou os valores das razões TS/SA e TS/ TI, corroborando os resultados de Martins et al (2008) que ao avaliar a razão TS/SA durante exercícios em CCF, entre os quais o push up nas bases estável e instável, semelhante ao OKP do presente estudo, não encontraram diferença significativa entre as superfícies.

Outro aspecto importante é o valor da razão $\mathrm{TS} / \mathrm{SA}$, que no presente estudo apresentou médias variando entre 0.40 e 0.69 , o que representa razões de ativação consideradas moderadas e divergem de estudos prévios (Ludewig et al., 2004; Martins et al., 2008) que encontraram razões TS/SA com valores considerados baixos, ou seja, próximos à 0.30 . Essas diferenças podem ser explicadas pelas diferenças da amostra, uma vez que Martins et al (2008) avaliaram homens sedentários e o presente estudo avaliou praticantes regulares de atividade física. Em relação à divergência com os resultados de Ludewig, Hoff, Osowski, Meschke, \& Rundquist (2004), a justificativa cabe às diferenças existentes na execução do exercício, o qual foi realizado de forma dinâmica e com incremento da fase de protração escapular, enquanto o presente estudo avaliou o exercício realizado de forma isométrica.

Para o grupo SIO foi observada também maior razão TS/TI comparada a TS/SA na maioria das situações avaliadas. Esses resultados sugerem que nestes indivíduos, pode existir de fato um desequilíbrio na ativação destes grupos musculares, seja devido à ativação excessiva do TS ou diminuição da atividade do TI e SA, que pode resultar principalmente, na diminuição do tipping e rotação superior da escápula, contribuindo para alterações na cinemática escapular (Ludewig \& Cook, 2000).

Apesar disso, o fato que melhor evidenciou a presença do desequilíbrio dos músculos estabi- lizadores da escápula nos voluntários do grupo SIO, foi justamente o aumento significativo da razão TS/SA com o incremento da superfície instável. Nesses exercícios, a presença da superfície instável impõe maior estresse ao sistema neuromuscular, fazendo com que aumente a demanda pela manutenção e controle da estabilização escapular, aquisições essas que deveriam ser garantidas principalmente pela sobreposição da ação do músculo SA em relação ao TS (Ludewig et al., 2004). No entanto, o observado no presente estudo foi justamente o contrário, visto que os valores das razões EMG aumentaram, indicando que proporcionalmente ocorreu um aumento da atividade do TS em relação ao SA.

Os resultados encontrados no presente estudo fornecem dados importantes para a elaboração de programas de reabilitação para indivíduos com SIO, baseados na possível correção do desequilíbrio muscular entre os estabilizadores escapulares, considerando não somente o SA, mas também o TI como importantes estabilizadores do ângulo inferior da escápula (Park \& Yoo, 2011). Nesse sentido, a inclusão de exercícios em CCF continua sendo opção interessante para o processo de treinamento neuromuscular, com ganhos moderados de força, visto que os níveis da atividade muscular ficaram próximos a faixa de $20 \%$ e $40 \%$ da CVM (DiGiovine, Jobe, Pink, \& Perry, 1992).

Além disso, o presente estudo abre um espaço para discussão sobre qual o melhor momento para a inserção da superfície instável no processo de reabilitação, visto que a resposta neuromuscular dos voluntários do grupo SIO divergiu do grupo controle e do que se era esperado. Apesar de se tratar de indivíduos fisicamente ativos, foi possível observar a presença de desequilíbrio nos músculos estabilizadores da escápula, o que talvez sugira a necessidade de se trabalhar, previamente à inserção da superfície instável, o fortalecimento seletivo dos músculos SA e TI em situações que a escápula esteja melhor estabilizada, a exemplo 
do exercício bench-press para o SA (Araújo et al., 2009; Tucci et al., 2011) , e exercícios em cadeia cinética aberta para o TI (McLean, Arlotta, \& Lo Vasco, 2011).

\section{Limitações e Estudos futuros}

A análise do sinal EMG foi realizada durante a realização dos exercícios de forma isométrica, não permitindo a generalização dos resultados para atividades dinâmicas. Além disso, no presente estudo não foi controlada a carga gerada em cada tarefa. No entanto, a realização de contrações isométricas permitiu uma análise mais confiável dos dados EMG, além disso, a proposta do estudo foi analisar exercícios que fazem parte das fases iniciais dos programas de reabilitação. A partir desses resultados, estudos futuros podem investigar o comportamento dos músculos durante a execução dinâmica desses exercícios, a fim de estabelecer evidências que suportem não somente sua inserção, mas também predigam o melhor momento da sua inclusão nos programas de reabilitação.

\section{CONCLUSÕES}

Os resultados do estudo evidenciaram diferentes respostas de ativação muscular nos indivíduos assintomáticos e com SIO frente a condições que alteram a estabilidade da base de apoio, uma vez que foram observadas maiores razões EMG nos exercícios em base instável, no grupo sintomático.

\footnotetext{
Agradecimentos:

À Fundação de Amparo à Ciência e Tecnologia do Estado de Pernambuco (FACEPE), pelo auxílio com a bolsa de iniciação científica.
}

\section{Conflito de Interesses:}

Nada declarado.

\section{Financiamento:}

Programa Institucional de Bolsas de Iniciação Científica PIBIC/FACEPE/CNPq - BIC-0366-4.08/11.

\section{REFERÊNCIAS}

Andrade, R., Araújo, R.C., Tucci, H.T., Martins, J., \& Oliveira, A.S. (2011). Coactivation of the shoulder and arm muscles during closed kinetic chain exercises on an unstable surface. Singapore Medical Journal, 52(1), 35-41.

Araújo, R.C., Andrade, R., Tucci, H.T., Martins, J., Grossi, D.B., \& Oliveira, A.S. (2009). Reliability of electromyographic amplitude values of the upper limb muscles during closed kinetic chain exercises with stable and unstable surfaces. Journal of Electromyography \& Kinesiology, 19(4), 685-694. doi:10.1016/j.jekelin.2007.11.014

Araújo, R.C., Andrade, R., Tucci, H.T., Martins, J., \& Oliveira, A.S. (2011). Shoulder Muscular Activity During Isometric Three-Point Kneeling Exercise on Stable and Unstable Surfaces. Journal of Applied Biomechanics, 27(3):192-196.

Cools, A.M., Dewitte, V., Lanszweert, F., Notebaert, D., Roets, A., Soetens, B.,...Witvrouw, E.E.(2007). Rehabilitation of scapular muscle balance: which exercises to prescribe? American Journal of Sports Medicine, 35(10), 1744-1751. doi: 10.1177/0363546507303560

De Morais Faria, C.D., Teixeira-Salmela, L.F., de Paula Goulart F.R, \& de Souza Moraes GF. (2008). Scapular muscular activity with shoulder impingement syndrome during lowering of the arms. Clinical Journal of Sport Medicine, 18(20), 130-136. doi: 10.1097/JSM.0b013e318160c05d

DiGiovine, N., Jobe, F., Pink, P., \& Perry, J. (1992). An electromyographic analysis of the upper extremity in pitching. Journal of Shoulder and Elbow Surgery, 1(1), 15-25. doi:10.1016/S10582746(09)80011-6

Dillman, C.J., Murray, T.A., \& Hintermeister, R.A. (1994). Biomechanical differences of open and closed chain exercises with respect to the shoulder. Journal of Sport Rehabilitation, 3(3), 228-238.

Hardwick, D.H., Beebe, J.A., McDonnell, M.K., \& Lang, C.E. (2006). A comparison of serratus anterior muscle activation during a wall slide exercise and other traditional exercises. Journal of Orthopaedic \& Sports Physical Therapy, 
36(12), 903-910. doi:10.2519/jospt.2006.2306

Hawkins, R.J., \& Kennedy, J.C. (1980). Impingement syndrome in athletes. American Journal of Sports Medicine, 8(3), 151-8. doi: $10.1177 / 036354658000800302$

Hermens, H.J., Freriks, B., \& Merletti, R.(1999). European recommendations for surface electromyography. Seniam deliverable 8- Roessingh research and development.

Hintermeinster, R.A., Lange, G.W., Schultheis, J.M., Bey, M.G., \& Hawkins, R.J. (1998). Electromyography activity and applied load during shoulder rehabilitation exercises using elastic resistance. American Journal of Sports Medicine, 26(2), 210-220.

Jobe, F.W., \& Moynes, D.R. (1982). Delineation of diagnostic criteria and a rehabilitation program for rotator cuff injuries. American Journal of Sports Medicine, 10(6), 336-9. doi: 10.1177/036354658201000602

Kendall, P.F., McCreary, E.K., \& Provance, P.G. (1995). Músculos: Provas e Funções (4a ed.). São Paulo: Manole.

Kibler, W.B. (1998). The role of the scapula in athletic shoulder function. American Journal of Sports Medicine, 26(2), 325-37.

Kibler, B., \& Livingston, W. (2001). Closed-Chain rehabilitation for upper and lower extremities. Journal of the American Academy of Orthopaedic Surgeon, 9(6), 412-421.

Kibler, W.B., Uhl, T.L., Maddux, J.W., Brooks, P.V., Zeller, B., \& McMullen, J. (2002). Qualitative clinical evaluation of scapular dysfunction: a reliability study. Journal of Shoulder and Elbow Surgery, 11(6), 550-6. doi:10.1067/ mse.2002.126766

Lehman, G.J., Gillas, D., \& Patel, U. (2008). An unstable support surface does not increase scapulothoracic estabilizing muscle activity during push up and push up plus exercises. Manual Therapy, 13(6), 500-506. doi:10.1016/j. math.2007.05.016

Lephart, S.M., \& Henry, T.J. (1996). The physiological basis for open and closed and kinetic chain rehabilitation for the upper extremity. Journal of
Sport Rehabilitation, 5(1), 71-87.

Ludewig, P.M., \& Cook, T.M. (2000). Alterations in shoulder kinematics and associated muscle activity in people with symptoms of shoulder impingement. Physical Therapy, 80(3), 276-291.

Ludewig, P.M., Hoff, M.S., Osowski, E.E., Meschke, S.A., \& Rundquist, P.J. (2004). Relative balance of serratus anterior and upper trapezius muscle activity during push-up exercises. American Journal of Sports Medicine, 32(2), 484-93.

Ludewig, P.M., \& Braman, J.P. (2011). Shoulder impingement: Biomechanical considerations in rehabilitation. Manual Therapy, 16(1), 33-39. doi: 10.1016/j.math.2010.08.004

Lukasiewicz, A.C., McClure, P., Michener, L., Pratt, N., \& Sennett, B. (1999) Comparison of 3-dimensional scapular position and orientation between subjects with and without shoulder impingement. Journal of Orthopaedic \& Sports Physical Therapy, 29(10), 574-86.

Martins, J., Tucci, H.T., Andrade, R., Araújo, R.C., Grossi, D.B., \& Oliveira, A.S. (2008). Electromyographic Amplitude Ratio of Serratus Anterior and Upper Trapezius Muscles During Modified Push-Ups and Bench Press Exercises. Journal of Strength Conditioning Research, 22(2), 477-484. doi: 10.1519/JSC.0b013e3181660748

McClure, P.W., Michener, L.A., Sennett, B.J., \& Karduna, A.R. (2001). Direct 3-dimensional measurement of scapular kinematics during dynamic movements in vivo. Journal of Shoulder and Elbow Surgery, 10(3), 269-77. doi: 10.1067/ mse.2001.112954

McLean, L., Arlotta, M., \& LoVasco, G. (2011). Selective recruitment of the lower fibers of the trapezius muscle. Journal of Electromyography \& Kinesiology, 21(3), 403-10. doi:10.1016/j. jekelin.2010.11.006

Neer, C.S. (1983). Impingement lesions. Clinical Orthopaedics and Related Research, 173, 70e7.

Oliveira, A.S., de Morais, C.M., \& de Brum, D.P. (2008). Activation of the shoulder and arm muscles during axial load exercises on a stable base of support and on a medicine ball. Journal of Electromyography \& Kinesiology, 18(3), 472-479. 
doi:10.1016/j.jelekin.2006.09.009

Park, S.Y., \& Yoo, W.G. (2011). Differential activation of parts of the serratus anterior muscle during push-up variations on stable and unstable bases of support. Jounal of Electromyography \& Kinesiology, 21 (5), 861-7. doi:10.1016/j. jelekin.2011.07.001

Phadke, V., Camargo, P.R., \& Ludewig, P.M. (2009). Scapular and rotator cuff muscle activity during arm elevation: a review of normal function and alterations with shoulder impingement. Revista Brasileira de Fisioterapia, 13(1), 1-9. doi: 10.1590/S1413-35552009005000012

Tucci, H.T., Ciol, M.A., de Araújo, R.C., de Andrade, R., Martins, J., McQuade, K.J., \& Oliveira, A.S. (2011). Activation of Selected Shoulder Muscles During Unilateral Wall and Bench Press Tasks Under Submaximal Isometric Effort. Journal of Orthopaedic \& Sports Physical Therapy, 41(7), 520-5. doi: 10.2519/jospt.2011.3418
Tucker, W.S., Campbell, B.M., Swartz, E.E., \& Armstrong. C;W. (2008). Electromyography of 3 scapular muscles: a comparative analysis of the cuff link device and a standard push-up. Journal of Athletic Training, 43 (5), 464-469.

Tucker, W.S., Armstrong, C.W., Gribble, P.A., Timmons, M.K., \& Yeasting, R.A. (2010). Scapular muscle activity in overhead athletes with symptoms of secondary shoulder impingement during closed chain exercises. Archives of Physical Medicine \& Rehabilitation, 91 (4), 550-6. doi:10.1016/j.apmr.2009.12.021

(c) EY-No Todo o conteúdo da revista Motricidade está licenciado sob a Creative Commons, exceto quando especificado em contrário e nos conteúdos retirados de outras fontes bibliográficas. 